\title{
Association between television viewing and the risk of metabolic syndrome in a community-based population Pei-Chia Chang1, Tsai-Chung Li ${ }^{* 2,3,4,5}$, Ming-Tsang $\mathrm{Wu}^{6}$, Chiu-Shong Liu ${ }^{7,8}$, Chia-Ing Li ${ }^{2}$, Ching-Chu Chen 7,8 , Wen-Yuan Lin ${ }^{7,8}$, Shin-Yuh Yang ${ }^{3}$ and Cheng-Chieh $\operatorname{Lin} * 2,5,7,8,9$
}

\begin{abstract}
Address: ${ }^{1}$ Administration Center, China Medical University Hospital, Taichung, Taiwan, ${ }^{2}$ Department of Medical Research, China Medical University Hospital, Taichung, Taiwan, ${ }^{3}$ Graduate Institute of Chinese Medicine Science, College of Chinese Medicine, China Medical University, Taichung, Taiwan, ${ }^{4}$ Biostatistics Center, China Medical University, Taichung, Taiwan, ${ }^{5}$ Institute of Health Care Administration, College of Health Science, Asia University, Taichung, Taiwan, ${ }^{6}$ Graduate Institute of Occupational Safety and Health, Kaohsiung Medical University, Taiwan, ${ }^{7}$ Department of Family Medicine, China Medical University Hospital, Taichung, Taiwan, ${ }^{8}$ Department of Family Medicine, College of Medicine, China Medical University, Taichung, Taiwan and ' $S$ School and Graduate Institute of Health Care Administration, College of Public Health, China Medical University, Taichung, Taiwan

Email: Pei-Chia Chang - lisa319.tw@yahoo.com.tw; Tsai-Chung Li* - tcli@mail.cmu.edu.tw; Ming-Tsang Wu - mingtsangwu@yahoo.com; Chiu-Shong Liu - liucs@ms14.hinet.net; Chia-Ing Li - a6446@www.cmuh.org.tw; Ching-Chu Chen - chingchu@ms15.hinet.net; WenYuan Lin -wylin@mail.cmu.edu.tw; Shin-Yuh Yang - yz123kimo@yahoo.com.tw; Cheng-Chieh Lin* - cmuh404@yahoo.com.tw

* Corresponding authors
\end{abstract}

Published: 3 June 2008

BMC Public Health 2008, 8:193 doi:10.1/86/147/-2458-8-193
Received: 28 October 2007

Accepted: 3 June 2008

This article is available from: http://www.biomedcentral.com/I47I-2458/8/I93

(C) 2008 Chang et al; licensee BioMed Central Ltd.

This is an Open Access article distributed under the terms of the Creative Commons Attribution License (http://creativecommons.org/licenses/by/2.0), which permits unrestricted use, distribution, and reproduction in any medium, provided the original work is properly cited.

\begin{abstract}
Background: As a result of metabolic syndrome becoming an important issue during recent decades, many studies have explored the risk factors contributing to its development. However, less attention has been paid to the risk associated with sedentary behavior, especially television viewing. This study examined the association between television viewing time and the risk of having metabolic syndrome in a population of Taiwanese subjects.
\end{abstract}

Methods: This community-based cross-sectional study included 2,353 subjects (I,I44 men and I,209 women) aged 40 and over from October, 2004 to September, 2005. Information about the time spent watching TV was obtained using a self-administered questionnaire. The definition of metabolic syndrome was according to the Third Report of the National Cholesterol Education Program's Adult Treatment Panel modified for Asians.

Results: Compared to subjects who viewed TV $<14 \mathrm{hr} /$ week, those who viewed TV $>20 \mathrm{hr} /$ week had a 1.50 -fold $(95 \%$ confidence intervals $(\mathrm{Cl})$ : I. $10,2.03)$ risk for men and a 1.93 -fold $(95 \% \mathrm{Cl}$ : I.37, 2.7I) risk for women of having metabolic syndrome, after adjusting for physical activity and other covariates. Stratifying by the three categories of total activity levels, TV viewing time $>20 \mathrm{hr} /$ week was found to still hold a significant risk for having metabolic syndrome in the lowest of the three categories of total activity level for men and in all three categories of total activity level for women.

Conclusion: The findings suggest that TV viewing is an independent risk factor associated with metabolic syndrome in Taiwanese people. 


\section{Background}

There is a high worldwide prevalence of metabolic syndrome, with the rate in each country depending on the particular diagnostic definition and the ethnicity of the population. The prevalence of metabolic syndrome has been found to be 20-32 percent for Caucasians [1-5], but only 15-24 percent of the Taiwanese population has been diagnosed [6-8]. Previous studies have shown that individuals with metabolic syndrome have an increased risk to have type 2 diabetes mellitus (DM), cardiovascular disease (CVD), hypertension and stroke, etc. [9-20]. Because of its high prevalence rate among the general population and the fact that it inevitably results in type-2 DM and $\mathrm{CVD}$, the syndrome has a great impact on public health.

As a result of metabolic syndrome becoming an important issue during recent decades, many studies have explored the risk factors contributing to its development. Although the syndrome has often been found to be associated with socio-demographic and lifestyle factors [5,21-26], less attention has been paid to the risk associated with sedentary behavior, especially television (TV) viewing, that has been reported to increase the risk of obesity and coronary heart disease (CHD) [27-33]. A few epidemiological studies have examined the effect of TV viewing on the chances of having metabolic syndrome and found that a prolonged viewing time of at least 2 hours per day was responsible for increasing the risk of having metabolic syndrome 1.74-3.30-fold [34-37]. However, all these studies were conducted on Caucasian populations, and it may be that Asian populations have different risk factors for metabolic syndrome. Therefore, in our study, we examined the association between TV viewing time and the presence of metabolic syndrome in a Taiwanese population as defined by the Third Report of the National Cholesterol Education Program's Adult Treatment Panel modified for Asians [38].

\section{Methods}

\section{Population and participants}

This is a community-based cross-sectional study. The target population consisted of people aged 40 and over who were residents in Taichung City, Taiwan, in October 2004. A total of 363,543 residents, or 4.09 percent of all Taiwanese residents aged $\geq 40$ years, lived in this area during the time of our study, and the sampling frame of which was all the family records held by the Bureau of Households in Taichung City. A two-stage sampling procedure was designed to draw participants, with a sampling rate proportional to size within each stage. In the first stage of sampling, the sampling unit was Li (blocks of household units) and the selection probability for Li was set at 0.125 . A total of 39 Lis were randomly selected from each city district (a total of 8 city districts). In the second stage, 4,280 individuals were selected by randomly selecting as close as 110 persons as possible from each sample Li [3941].

Of the 4,280 subjects selected for our sample, 750 were excluded because of death (18), hospitalization or imprisonment (14), living abroad (39), moving away from the original address (411), living outside of the study area (7), not being at home during 3 attempts to visit them (202) or other reasons (59). Of the 3,530 remaining subjects, 2,359 were willing to participate in this study. However, because 6 subjects did not provide information about their TV viewing habits, 2,353 subjects $(1,144$ men and 1,209 women) were included in the final analysis. The age distribution among the non-participants was 47.6 percent (40-49 years), 25.5 percent (50-59 years), 13.8 percent (60-69 years) and 13.2 percent ( $\geq 70$ years), and this was comparable with that of the participants: 43.9 percent ( $40-49$ years), 30.9 percent (50-59 years), 14.8 percent (60-69 years) and 10.4 percent ( $\geq 70$ years). The study was approved by the Instituted Review of Board of China Medical University Hospital. Written informed consent was obtained from all participants.

All subjects who were eligible for participation were asked to complete a self-reported questionnaire and this was followed by a series of physical examinations in the hospital (China Medical University \& Hospital) between October, 2004 and September, 2005, during which participants were also required to provide a blood sample. The questionnaire required participants to provide socio-demographic characteristics and lifestyle details, such as smoking, drinking, leisure and occupational activity, and time spent on watching TV every week. Information regarding time for watching TV was obtained using the open question "On average, how many hours a day (or a week) do you spend on watching TV?" The physical activity for leisure time of a participant was determined by the different kinds of physical activities each person participated in, the average time spent on this activity per occasion and the total time per week the person spent performing each activity during the past year. The checklist of leisure-time activity contained 26 items including ball games, martial arts, aerobic sports, dancing and others. We assigned a metabolic equivalent (MET) to each kind of activity and then calculated each participant's leisure-time activity by the formula of activity: MET× averaged duration of spending on this activity $(\mathrm{hr}) \times$ total number of times a week. The occupational activity status was determined by 4 levels according to the degree of manual labor involved: seated most of the time, light, moderate, and heavy activity and then assigned a MET to each. The occupational activity status was then calculated using the formula: manual labor intensity level $\times$ working time a day $(\mathrm{hr}) \times$ working days a week. The adjusted carbohydrate intake by total energy intake was computed by 
dividing caloric intake in the form of carbohydrates by total energy intake [42], and was categorized as being either moderate ( $40-60$ percent) or high ( $>60$ percent) [5].

\section{Anthropometric measurement and laboratory examination}

Body height and weight were measured using an autoanthropometer (Super-view, HW-666, Taipei, Taiwan) with participants wearing light clothing and no shoes. The waist circumference was measured midway between the iliac and the costal margin, with the subjects in standing position and the hip circumference was measured at its maximum in order to calculate the waist-to-hip ratio. The blood pressure was measured using participants' right arm after they had been resting in a seated position for $20 \mathrm{~min}$ utes. The means of two systolic and two diastolic blood pressures were recorded.

Blood was drawn from an antecubital vein in the morning after a 10-to-12-hour overnight fasting and was sent for analysis within four hours of blood. A one-spot urine specimen was also collected. Biochemical markers such as serum HDL-cholesterol, triglyceride, and fasting glucose as well as urine albumin and creatinine were analyzed using a biochemical auto-analyzer (Beckman Coulter, Lx20, USA) at the Department of Clinical Laboratory of China Medical University Hospital.

\section{Definition of metabolic syndrome}

A modification for Asians of the Third Report of the National Cholesterol Education Program's Adult Treatment Panel [38] was used to define metabolic syndrome. According to the report, metabolic syndrome involves three or more of the following abnormalities: a fasting plasma glucose level $\geq 110 \mathrm{mg} / \mathrm{dl}$, a serum triglyceride level $\geq 150 \mathrm{mg} / \mathrm{dl}$, a serum HDL-cholesterol level $<40 \mathrm{mg} /$ $\mathrm{dl}$ for men and $<50 \mathrm{mg} / \mathrm{dl}$ for women, a blood pressure level $\geq 130 / 85 \mathrm{mmHg}$, or a waist circumference $>90 \mathrm{~cm}$ for Asian men and $>80 \mathrm{~cm}$ for Asian women. However, the modification is regarding the cut-offs for abdominal obesity. Subjects who took medication for hyperglycemia, hypertension, hypercholesterolemia or hypertriglyceridemia, were still considered to have abnormal laboratory data.

\section{Statistical analysis}

Mean (standard deviation, SD) and number (percentage) of demographic characteristics and other variables categorized by status of TV viewing time status $(<14,14-20$, and $>20 \mathrm{hr}$ /week) were compared using ANOVA or chi-square statistics for men and women. Four age categories were created using 10 year intervals $(40-49,50-59,60-69, \geq$ $70 \mathrm{yrs}$ ) while three categories were defined based on participants' education level: $<9,9-12$, and $>12$ yrs. Three economic status categories were created according to participants' household income per month: < 40,000, 40,000-100,000, and > 100,000 Taiwanese dollars. Smoking and drinking were categorized as never, former and current and the carbohydrate intake was either moderate or high. Leisure-time activity was dichotomized to either $<19.5$ or $\geq 19.5$ MET-hour per week (the first and second tertiles were combined due to only few individuals in the second tertile); the occupational activity status was categorized by the tertiles of number of metabolic equivalent hours per week: $<40,40-83$ or $>83$ MET-hour per week. The total activity was obtained by summing the leisure-time and occupational activities and then divided into three categories by the tertiles: $<54,54-103.3$ or $>$ 103.3 MET-hour per week.

Spearman correlation statistics were used to determine whether there was any correlation between certain continuous variables. Multivariate unconditional logistic regressions were used to assess the association between metabolic syndrome and the length of time spent on viewing TV (baseline: $<14 \mathrm{hr} /$ week) after adjusting for other covariates. Covariates in the final models included age, level of education, household income, occupational activity status, and smoking which were significant or marginally significant in the univariate analyses.

In order to examine the joint association of total activity and TV viewing time, we examined the association between the TV viewing time and the risk of having metabolic syndrome stratified by categories of total activity in men and women.

The responses given by 241 men and 204 women indicated that they had a history of physician-diagnosed heart diseases, stroke, or cancers, but when we excluded them and re-analyzed the data, we found the results to be similar. We therefore presented our findings without exclusion. All data was analyzed using Statistical Analysis Software. Odds ratios and 95 percent confidence intervals (CI) were calculated and significance levels of $\mathrm{p}<0.05$ were reported.

\section{Results}

Table 1 shows the socio-demographic characteristics according to groups of TV viewing time stratified by gender. In men, TV viewing time varied significantly depending on age, education level, household income, cigarette smoking, occupation activity status and their total activity status $(\mathrm{p}$-value $=0.0042$ to $<0.0001)$. For women the results were similar (p-value $=0.0004$ to $<0.0001)$ with the exception of cigarette smoking and total activity status. The physical and biochemical variables are summarized in Table 2 according to groups of TV viewing time stratified by gender. Except for the diastolic BP in men and 
Table I: Distributions of sociodemographic characteristics according to status of TV viewing time stratified by gender

\begin{tabular}{|c|c|c|c|c|c|c|c|c|}
\hline \multirow[b]{3}{*}{ Variables } & \multicolumn{4}{|c|}{ Men $(n=1144)$} & \multicolumn{4}{|c|}{ Women $(n=1209)$} \\
\hline & \multicolumn{3}{|c|}{ TV viewing time $n(\%)$} & \multirow[b]{2}{*}{ p-value ${ }^{b}$} & \multicolumn{3}{|c|}{ TV viewing time $n(\%)$} & \multirow[b]{2}{*}{ p-value ${ }^{b}$} \\
\hline & $\begin{array}{c}<14 \\
(n=329)\end{array}$ & $\begin{array}{c}14-20 \\
(n=343)\end{array}$ & $\begin{array}{c}>20 \\
(n=472)\end{array}$ & & $\begin{array}{c}<14 \\
(n=342)\end{array}$ & $\begin{array}{c}14-20 \\
(n=306)\end{array}$ & $\begin{array}{c}>20 \\
(n=561)\end{array}$ & \\
\hline Age $(y r s)^{a}$ & $56.08(0.82)$ & $57.47(0.80)$ & $61.15(0.68)$ & $<.0001 * * * *$ & $53.06(0.69)$ & $54.46(0.73)$ & $57.01(0.54)$ & $<.0001 * * *$ \\
\hline Education Level & & & & $0.0027^{* *}$ & & & & $<.0001 * * *$ \\
\hline$<9$ & $88(26.75)$ & $92(26.82)$ & $149(3 \mid .57)$ & & $120(35.09)$ & $|3|(42.81)$ & $280(49.91)$ & \\
\hline $9-12$ & $|4|(42.86)$ & $167(48.69)$ & $236(50.00)$ & & $152(44.44)$ & $129(42.16)$ & $237(42.25)$ & \\
\hline$>12$ & $100(30.40)$ & $84(24.49)$ & $87(18.43)$ & & $70(20.47)$ & $46(15.03)$ & $44(7.84)$ & \\
\hline Marriage status & & & & 0.6908 & & & & 0.6338 \\
\hline married & $286(86.93)$ & $305(88.92)$ & $418(88.56)$ & & $259(75.73)$ & $24 I(78.76)$ & $429(76.47)$ & \\
\hline others & $43(13.07)$ & $38(11.08)$ & $54(I I .44)$ & & $83(24.27)$ & $65(21.24)$ & $132(23.53)$ & \\
\hline $\begin{array}{l}\text { Household } \\
\text { income } \\
\text { (Taiwanese } \\
\text { dollars/month) }\end{array}$ & & & & $0.0042^{* *}$ & & & & $0.0002^{* * *}$ \\
\hline$<40,000$ & |44(43.77) & $155(45.19)$ & $260(55.08)$ & & $154(45.03)$ & | $43(46.73)$ & $304(54.19)$ & \\
\hline $40,000-100,000$ & I36(41.34) & $147(42.86)$ & $170(36.02)$ & & $138(40.35)$ & 134(43.79) & $224(39.93)$ & \\
\hline$>100,000$ & $49(\mid 4.89)$ & $4 I(I I .95)$ & $42(8.90)$ & & $50(14.62)$ & $29(9.48)$ & $33(5.88)$ & \\
\hline Smoking & & & & $0.0007^{* * *}$ & & & & 0.3452 \\
\hline never & 184(55.93) & $|7|(49.85)$ & $197(41.74)$ & & $327(95.61)$ & $296(96.73)$ & $529(94.30)$ & \\
\hline former & $59(17.93)$ & $71(20.70)$ & $132(27.97)$ & & $2(0.58)$ & $3(0.98)$ & $10(1.78)$ & \\
\hline current & $86(26.14)$ & $101(29.45)$ & $143(30.30)$ & & $13(3.80)$ & $7(2.29)$ & $22(3.92)$ & \\
\hline Drinking & & & & 0.0536 & & & & 0.6475 \\
\hline never & $190(57.75)$ & $\mid 88(54.8 \mid)$ & $24 I(5 I .96)$ & & $302(88.30)$ & $274(89.54)$ & $496(88.4 I)$ & \\
\hline former & $36(10.94)$ & $35(10.20)$ & $37(7.84)$ & & $5(1.46)$ & $\mathrm{I}(0.33)$ & $8(1.43)$ & \\
\hline current & $103(31.31)$ & $120(34.99)$ & $194(41.10)$ & & $35(10.23)$ & $31(10.13)$ & $57(10.16)$ & \\
\hline $\begin{array}{l}\text { Adjusted } \\
\text { carbohydrate } \\
\text { intake }\end{array}$ & & & & 0.6862 & & & & 0.3142 \\
\hline moderate & $4(1.22)$ & $3(0.87)$ & $3(0.64)$ & & $6(1.75)$ & $6(1.96)$ & $18(3.21)$ & \\
\hline high & $325(98.78)$ & $340(99.13)$ & $469(99.36)$ & & $336(98.25)$ & $300(98.04)$ & $543(96.79)$ & \\
\hline $\begin{array}{l}\text { Leisure-time } \\
\text { activity (MET- } \\
\text { hour/week) }\end{array}$ & & & & 0.7134 & & & & 0.5496 \\
\hline$<19.5$ & $210(63.83)$ & $209(60.93)$ & $298(63.14)$ & & $229(66.96)$ & $217(70.92)$ & $388(69.16)$ & \\
\hline$\geq 19.5 \leq$ & $119(36.17)$ & 134(39.07) & $174(36.86)$ & & $113(33.04)$ & $89(29.08)$ & $173(30.84)$ & \\
\hline $\begin{array}{l}\text { Occupational } \\
\text { activity (MET- } \\
\text { hour/week) }\end{array}$ & & & & $<.0001 * * *$ & & & & $0.0004^{* * * *}$ \\
\hline$<40$ & $97(29.48)$ & $102(29.74)$ & $208(44.07)$ & & $89(26.02)$ & $67(21.90)$ & $191(34.05)$ & \\
\hline $40-83$ & $124(37.69)$ & $115(33.53)$ & $124(26.27)$ & & $138(40.35)$ & $116(37.91)$ & $169(30.12)$ & \\
\hline$>83$ & $108(32.83)$ & $126(36.73)$ & $140(29.66)$ & & $115(33.63)$ & $123(40.20)$ & $201(35.83)$ & \\
\hline $\begin{array}{l}\text { Total activity } \\
\text { status (MET- } \\
\text { hour/week) }\end{array}$ & & & & $<.0001 * * *$ & & & & 0.1689 \\
\hline $0-54$ & $90(27.36)$ & $105(30.61)$ & $200(42.37)$ & & $98(28.65)$ & $86(28.10)$ & 192(34.22) & \\
\hline $54-103.3$ & $120(36.47)$ & $105(30.61)$ & $137(29.03)$ & & $128(37.43)$ & $103(33.66)$ & $188(33.51)$ & \\
\hline $103.3<$ & $119(36.17)$ & $133(38.78)$ & $135(28.60)$ & & $116(33.92)$ & $117(38.24)$ & $|8|(32.26)$ & \\
\hline
\end{tabular}

aValues are expressed as means (standard deviation).

bFor the difference among groups of TV viewing time.

$*_{p}<0.05$ (bolded);** $<0.01$ (bolded);***p $<0.001$ (bolded)

MET, metabolic equivalent. 
Table 2: Comparisons of physical and biochemical examinations among groups of TV viewing time stratified by gender

\begin{tabular}{|c|c|c|c|c|c|c|c|c|}
\hline \multirow[b]{3}{*}{ Variables } & \multicolumn{4}{|c|}{ Men $(n=1144)$} & \multicolumn{4}{|c|}{ Women $(n=1209)$} \\
\hline & \multicolumn{3}{|c|}{ TV viewing time means (standard deviation) } & \multirow[b]{2}{*}{ p-value ${ }^{a}$} & \multicolumn{3}{|c|}{ TV viewing time means (standard deviation) } & \multirow[b]{2}{*}{ p-value ${ }^{a}$} \\
\hline & $\begin{array}{c}<14 \\
(n=329)\end{array}$ & $\begin{array}{c}14-20 \\
(n=343)\end{array}$ & $\begin{array}{c}>20 \\
(n=472)\end{array}$ & & $\begin{array}{c}<14 \\
(n=342)\end{array}$ & $\begin{array}{c}14-20 \\
(n=306)\end{array}$ & $\begin{array}{c}>20 \\
(n=561)\end{array}$ & \\
\hline BMI $\left(\mathrm{kg} / \mathrm{m}^{2}\right)$ & $24.35(0.21)$ & $24.76(0.21)$ & $25.07(0.18)$ & 0.0066 & $23.13(0.22)$ & $23.85(0.24)$ & $24.36(0.17)$ & $<.0001 * * *$ \\
\hline $\begin{array}{l}\text { Waist } \\
\text { circumference } \\
\text { (cm) }\end{array}$ & $84.90(0.59)$ & $86.11(0.57)$ & $87.52(0.49)$ & $0.0001 * * *$ & $74.61(0.58)$ & $76.52(0.61)$ & $78.04(0.45)$ & $<.000 I^{* * * *}$ \\
\hline $\begin{array}{l}\text { Systolic BP } \\
(\mathrm{mmHg})\end{array}$ & $136.72(1.39)$ & $137.44(1.36)$ & $141.40(1.16)$ & $0.0022^{* *}$ & |27.3| (|.49) & $132.18(1.57)$ & $136.13(1.16)$ & $<.000 I^{* * *}$ \\
\hline $\begin{array}{l}\text { Diastolic BP } \\
(\mathrm{mmHg})\end{array}$ & $81.84(0.77)$ & $82.36(0.76)$ & $83.26(0.65)$ & 0.2085 & $72.92(0.81)$ & $75.32(0.85)$ & $77.12(0.63)$ & $<.0001 * * *$ \\
\hline $\begin{array}{l}\text { Fasting blood } \\
\text { glucose (mg/dl) }\end{array}$ & $103.07(1.98)$ & $103.63(1.94)$ & $110.16(1.66)$ & $0.0006^{* * *}$ & 97.08 (1.77) & $97.81(1.88)$ & $104.94(1.39)$ & $<.0001 * * *$ \\
\hline $\begin{array}{l}\text { HDL- } \\
\text { cholesterol } \\
\text { (mg/dl) }\end{array}$ & $41.65(0.74)$ & $4 I .4 \mid(0.72)$ & $41.47(0.61)$ & 0.9548 & $51.22(0.84)$ & $49.90(0.89)$ & $49.81(0.66)$ & 0.2347 \\
\hline $\begin{array}{l}\text { Triglyceride } \\
\text { (mg/dl) }\end{array}$ & 125.64 (7.52) & $130.36(7.37)$ & 148.04 (6.28) & $0.0102 *$ & $92.63(4.61)$ & $102.36(4.87)$ & I I 8.68 (3.60) & $<.0001^{* * *}$ \\
\hline
\end{tabular}

aFor the difference among groups of TV viewing time.

$*_{p}<0.05$ (bolded); **p $<0.0$ I (bolded); ***p $<0.001$ (bolded).

BMI, body mass index; BP, Blood pressure; HDL, high density lipoprotein.

the serum HDL levels in both men and women, an increase in the time spent viewing TV, resulted in higher mean BMI levels, a larger waist circumference and higher systolic BP, serum fasting glucose and triglyceride levels.

The Spearman correlation coefficients between age, leisure-time activity, occupational activity, TV viewing time and adjusted carbohydrate intake ranged from -0.32 to 0.23 , which were either weak or negligible (Table 3 ). The correlation between total activity with age and adjusted carbohydrate intake remained weak (Spearman correlation coefficients $=-0.23$ and -0.03 ). Therefore, there was not a strong multi-co-linearity between these covariates. On the contrary, the Spearman correlation coefficient between total activity and occupation activity was 0.92 , indicating there exists collinearity between them. The Spearman correlation coefficients between TV viewing time with leisure-time activity, occupational activity, and total activities were weak (ranging from -0.04 to -0.09 ), implying that sedentary behavior such as TV viewing was distinct behavior from physical activity.

Compared to subjects who viewed TV $<14 \mathrm{hr} /$ week, those who viewed TV $>20 \mathrm{hr} /$ week, men and women respectively had a 1.50 -fold (95 percent CI: $1.10,2.03)$ and 1.93-fold (95 percent CI: 1.37, 2.71) chance of having metabolic syndrome, after adjusting for age, educational level, household income, occupational activity, and ciga-

Table 3: Spearman correlation coefficients among age, variables for physical activity, TV viewing time, and adjusted carbohydrate intake.

Leisure-time activity Occupation activity Total activity TV viewing time Adjusted carbohydrate

\begin{tabular}{|c|c|c|c|c|c|}
\hline Age & $0.23^{* * *}$ & $-0.32 * * *$ & $-0.23 * * *$ & $0.17^{* * *}$ & $0.06 *$ \\
\hline Leisure-time activity & 1.00 & $-0.14^{* * *}$ & $0.18 * * *$ & -0.04 & $-0.09 * * *$ \\
\hline Occupation activity & - & 1.00 & $0.92 * * *$ & $-0.08^{*}$ & -0.01 \\
\hline Total activity & - & - & 1.00 & $-0.09 * * *$ & -0.03 \\
\hline TV viewing time & - & - & - & 1.00 & -0.01 \\
\hline $\begin{array}{l}\text { Adjusted carbohydrate } \\
\text { intake }\end{array}$ & - & - & - & - & 1.00 \\
\hline
\end{tabular}

$*_{p}<0.05$ (bolded).

$* * p<0.01$ (bolded).

$* * * p<0.001$ (bolded) 
Table 4: Relationship between TV viewing time and other co-variates and metabolic syndrome risk by gender

\begin{tabular}{|c|c|c|c|c|c|c|c|c|}
\hline \multirow[b]{2}{*}{ Variables } & \multicolumn{4}{|c|}{ Men $(n=1144)$} & \multicolumn{4}{|c|}{ Women $(n=1209)$} \\
\hline & $\begin{array}{c}\text { MS }^{a} \\
(n=431)\end{array}$ & $\begin{array}{l}\text { Non-MSa } \\
(n=7 \mid 3)\end{array}$ & $\begin{array}{l}\text { Crude OR } \\
(95 \% \mathrm{Cl})\end{array}$ & $\begin{array}{l}\text { Adjusted OR } \\
(95 \% \mathrm{Cl})\end{array}$ & $\begin{array}{c}M^{a} \\
(n=352)\end{array}$ & $\begin{array}{l}\text { Non-MS } \\
(n=857)\end{array}$ & $\begin{array}{c}\text { Crude OR } \\
(95 \% \mathrm{Cl})\end{array}$ & $\begin{array}{c}\text { Adjusted OR } \\
(95 \% \mathrm{Cl})\end{array}$ \\
\hline \multicolumn{9}{|c|}{ TV viewing time (hours/week) } \\
\hline$<14$ & $108(32.8)$ & $221(67.2)$ & 1.00 & 1.00 & $66(19.3)$ & $276(80.7)$ & 1.00 & 1.00 \\
\hline $14-20$ & $111(32.4)$ & $232(67.6)$ & $\begin{array}{l}\text { 0.98(0.7II, } \\
\quad I .35)\end{array}$ & $\begin{array}{c}0.96(0.69 \\
\text { I.33) }\end{array}$ & $80(26.1)$ & $226(73.9)$ & $\begin{array}{l}\text { I. } 48(1.02 \\
2.14)\end{array}$ & $\begin{array}{l}\text { I.38(0.93, } \\
2.05)\end{array}$ \\
\hline$>20$ & $212(44.9)$ & $260(55.1)$ & $\begin{array}{l}1.67(1.25 \\
2.24)\end{array}$ & $\begin{array}{l}1.50(1.10 \\
2.03)\end{array}$ & $206(36.7)$ & $355(63.3)$ & $\begin{array}{c}2.43(1.76 \\
3.34)\end{array}$ & $\begin{array}{l}1.93(1.37 \\
2.7 I)\end{array}$ \\
\hline \multicolumn{9}{|l|}{ Age (yrs) } \\
\hline $40-49$ & $110(32.6)$ & $227(67.4)$ & 1.00 & 1.00 & $61(13.3)$ & $397(86.7)$ & 1.00 & 1.00 \\
\hline $50-59$ & $119(35.0)$ & $221(65.0)$ & $\begin{array}{l}\text { I.II }(0.8 \mathrm{I} \\
\mathrm{I} .53)\end{array}$ & $\begin{array}{l}\text { I.02(0.73 } \\
\text { I.42) }\end{array}$ & $120(31.0)$ & $267(69.0)$ & $\begin{array}{c}2.93(2.07 \\
4.13)\end{array}$ & $\begin{array}{c}2.52(1.75 \\
3.62)\end{array}$ \\
\hline $60-69$ & $90(43.3)$ & $118(56.7)$ & $\begin{array}{l}1.57(1.10 \\
2.25)\end{array}$ & $\begin{array}{l}\text { I.34(0.9I, } \\
1.97)\end{array}$ & $105(43.8)$ & $135(56.2)$ & $\begin{array}{c}5.06(3.49 \\
7.34)\end{array}$ & $\begin{array}{c}3.79(2.5 \mathrm{I} \\
5.72)\end{array}$ \\
\hline$\geq \mathbf{7 0}$ & $1 \mathrm{I} 2(43.2)$ & $147(56.8)$ & $\begin{array}{l}1.57(1.13 \\
2.20)\end{array}$ & $\begin{array}{l}1.20(0.81 \\
\text { I.79) }\end{array}$ & $66(53.2)$ & $58(46.8)$ & $\begin{array}{l}7.4 I(4.75 \\
\quad 11.54)\end{array}$ & $\begin{array}{l}5.31(3.22 \\
8.77)\end{array}$ \\
\hline \multicolumn{9}{|c|}{ Educational level } \\
\hline$<9$ & $139(42.3)$ & 190(57.7) & 1.00 & 1.00 & $2 \mid 8(4 I .1)$ & $313(58.9)$ & 1.00 & 1.00 \\
\hline $9-12$ & $206(37.9)$ & $338(62.1)$ & $\begin{array}{l}0.83(0.63 \\
\quad I .10)\end{array}$ & $\begin{array}{c}0.97(0.72 \\
\text { I.3I) }\end{array}$ & $105(20.3)$ & $413(79.7)$ & $\begin{array}{c}0.37(0.28 \\
0.48)\end{array}$ & $\begin{array}{c}0.63(0.46 \\
0.86)\end{array}$ \\
\hline$>12$ & $86(31.7)$ & $185(68.3)$ & $\begin{array}{c}0.64(0.45 \\
0.89)\end{array}$ & $\begin{array}{l}0.81(0.54 \\
1.19)\end{array}$ & $29(18.1)$ & $131(81.9)$ & $\begin{array}{c}0.32(0.21 \\
0.49)\end{array}$ & $\begin{array}{l}0.67(0.41 \\
\text { I.II) }\end{array}$ \\
\hline \multicolumn{9}{|c|}{ Household income (Taiwanese thousand dollars/month) } \\
\hline$<40$ & $237(42.4)$ & $322(57.6)$ & 1.00 & 1.00 & $214(35.6)$ & $387(64.4)$ & 1.00 & 1.00 \\
\hline $40-100$ & $149(32.9)$ & $304(67.1)$ & $\begin{array}{l}0.67(0.5 \mathrm{I} \\
0.86)\end{array}$ & $\begin{array}{c}0.79(0.59 \\
1.06)\end{array}$ & $116(23.4)$ & $380(76.6)$ & $\begin{array}{l}0.55(0.42 \\
0.72)\end{array}$ & $\begin{array}{l}0.82(0.61 \\
I .10)\end{array}$ \\
\hline$>100$ & $45(34.1)$ & $87(65.9)$ & $\begin{array}{c}0.70(0.47 \\
\quad 1.05)\end{array}$ & $\begin{array}{c}0.89(0.56 \\
1.39)\end{array}$ & $22(19.6)$ & $90(80.4)$ & $\begin{array}{c}0.44(0.27 \\
0.73)\end{array}$ & $\begin{array}{l}0.82(0.47 \\
\quad I .43)\end{array}$ \\
\hline \multicolumn{9}{|c|}{ Occupation activity (MET-hour) } \\
\hline$<40$ & $178(41.3)$ & $229(56.3)$ & 1.00 & 1.00 & $122(35.2)$ & $225(64.8)$ & 1.00 & 1.00 \\
\hline $40-83$ & $137(37.7)$ & $226(62.3)$ & $\begin{array}{l}0.78(0.58 \\
\quad I .04)\end{array}$ & $\begin{array}{l}0.95(0.69 \\
\text { I.29) }\end{array}$ & $105(24.8)$ & $318(75.2)$ & $\begin{array}{c}0.61(0.45 \\
0.83)\end{array}$ & $\begin{array}{c}\text { 0.94(0.67, } \\
\text { I.33) }\end{array}$ \\
\hline$>83$ & $116(31.0)$ & $258(69.0)$ & $\begin{array}{c}0.58(0.43 \\
0.78)\end{array}$ & $\begin{array}{c}0.64(0.46 \\
0.88)\end{array}$ & $125(28.5)$ & $3 \mid 4(7 \mid .5)$ & $\begin{array}{c}0.73(0.54 \\
0.99)\end{array}$ & $\begin{array}{l}\text { I.08(0.77, } \\
\text { I.52) }\end{array}$ \\
\hline \multicolumn{9}{|c|}{ Cigarette smoking } \\
\hline Never & $189(34.2)$ & $363(65.8)$ & 1.00 & 1.00 & $330(28.7)$ & $822(71.3)$ & 1.00 & 1.00 \\
\hline former & $103(39.3)$ & $159(60.7)$ & $\begin{array}{l}\text { I.24(0.92, } \\
\text { I.69) }\end{array}$ & $\begin{array}{c}\text { I. } 14(0.83 \\
1.56)\end{array}$ & $9(60.0)$ & $6(40.0)$ & $\begin{array}{c}3.74(1.32 \\
10.58)\end{array}$ & $\begin{array}{c}4.67(1.49 \\
14.69)\end{array}$ \\
\hline Current & $139(42.1)$ & $191(57.9)$ & $\begin{array}{c}1.40(1.06 \\
1.85)\end{array}$ & $\begin{array}{c}1.42(1.06 \\
1.92)\end{array}$ & $13(3 \mid .0)$ & $29(69.0)$ & $\begin{array}{l}1.12(0.57 \\
2.18)\end{array}$ & $\begin{array}{c}\text { I.35(0.66, } \\
2.77)\end{array}$ \\
\hline
\end{tabular}

aValues are expressed as $\mathrm{n}$ (percentages); OR, odds ratio; $\mathrm{Cl}$, confidence interval.

rette smoking habits (Table 4 ). In contrast, we found no such significant relationship when we compared the 14$20 \mathrm{hr} /$ week TV viewing group with the $<14 \mathrm{hr} /$ week TV viewing group. Other covariates, including age in women and cigarette smoking in both men and women, were positively and significantly associated with the risk of having metabolic syndrome. In contrast, occupational activity and educational level were negatively and significantly associated with the risk of both men and women having metabolic syndrome, after adjusting for other covariates (Table 4).

When we categorized participants according to their total activity and then compared TV viewing time in each category, we found that men with TV viewing time $>20 \mathrm{hr} /$ week in the lowest category of total activity had a 2.00fold (95 percent CI:1.16, 3.46) risk of having metabolic syndrome compared to those with TV viewing time $<14$ 

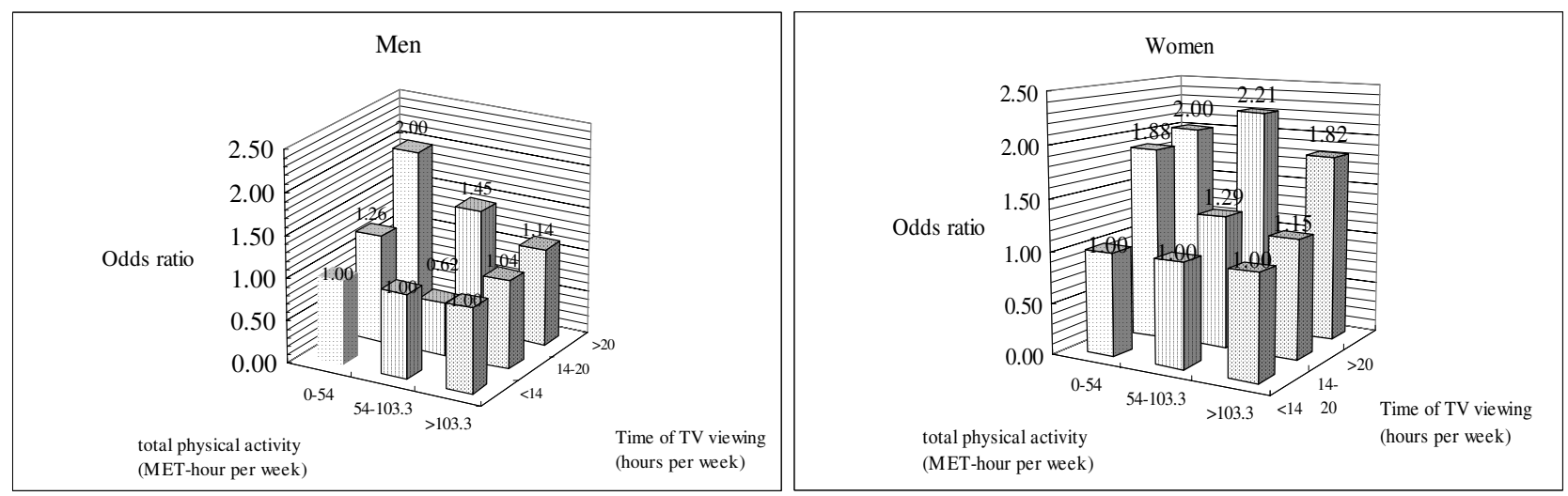

\section{Figure I}

Effect of time of television (TV) viewing and total physical activity to the risk of metabolic syndrome by gender adjusted for age, educational level, household income and cigarette smoking habits. Groups for time of TV viewing (hours per week)was classified as < I4, I4-20, $>20$ and total physical activity (MET-hr per week) as 0-54, 54-103.3, > 103.3.

$\mathrm{hr} /$ week in the lowest category of total activity (Figure 1). In addition, women with TV viewing time $>20 \mathrm{hr} /$ week had a significantly larger (1.82-2.21-fold) risk of having metabolic syndrome than those with TV viewing time < $14 \mathrm{hr} /$ week in all three categories of total activity (Figure 1). This result indicated that there was an association between risk of having metabolic syndrome and TV viewing time in women who had sufficient physical activity.

\section{Discussion}

In this study, we found that both men and women who on average spent more than 20 hours per week watching television had a significantly higher risk of having metabolic syndrome than those who spent less than 14 hours per week doing so. This effect was still significant, even after we adjusted for other covariates and created categories according to participants' level of total activity. To date, few epidemiological and clinical studies have examined the association between sedentary behaviors, such as TV viewing, and metabolic syndrome [34-37]. In 19951996, Gustat et al. (2002) conducted a community-based study in Bogalusa, Louisiana, to examine the relationship between physical activity and the risk of having insulin resistance. He recruited 409 African American and 1,011 White American participants, aged 20 to 38 years old, and used a questionnaire to measure the intensity of their physical inactivity as the sum of the time they spent watching TV and the time they spent using a computer. They divided the hours of inactivity into quintiles and found that subjects with more hours of inactivity ( $4^{\text {th }}$ and $5^{\text {th }}$ quintile) had a 1.9- (95 percent CI: 1.07, 3.25) and 2.5fold respective risk (95 percent CI: 1.49, 4.19) of having insulin resistance syndrome, compared to those in the lowest quintile ( $1^{\text {st }}$ quintile) of inactivity. They concluded that the length of inactivity is an important predictor of insulin resistance syndrome in young adults. Subsequently, two other published studies that were conducted on populations of American and French subjects had similar findings $[35,36]$. However, these studies did not consider the interrelationship between physical inactivity, dietary habits, and metabolic syndrome.

Recently, Dunstan and his co-workers analyzed the data of 6,162 Australian people aged 35 years or older who had participated in the 1999-2000 Australian Diabetes, Obesity and Lifestyle Study [37]. Only individuals without type 2 diabetes, self-reported angina, stroke and myocardial infarction, or those taking medications for hypertension or dyslypidemia were included. They found that for each 1 -hour increase in TV viewing time per day, there was a 21 percent $(\mathrm{p}=0.07)$ and 26 percent $(\mathrm{p}=0.0001)$ respective increase in the prevalence of metabolic syndrome in men and women, after taking into account physical activity, dietary habits, and other covariates. However, all these studies were conducted on Caucasian populations. To our knowledge, ours is the first study to observe that TV viewing is a risk factor for metabolic syndrome for an Asian population, in particular a Taiwanese population.

By which independent mechanism can the time spent viewing TV explain the association with metabolic syndrome? Firstly, TV viewing may be highly associated with poor eating habits, particularly the consumption of junk food and snacks while watching TV [43]. Although our study did not include detailed information about TV viewing and eating habits, we did have information about the daily averaged dietary intake. Even after providing for dietary intake and other covariates, we still found a signifi- 
cant positive association between TV viewing and the risk of having metabolic syndrome, an association that cannot be explained in full by the effect of poor eating habits while watching TV.

Secondly, previous studies have shown that there is an inverse relationship between metabolic syndrome and physical activity [34-37,44-47]. Due to modest inverse correlation between physical activity and physical inactivity (or sedentary behavior), the effect of the length time spent watching TV on the risk of having metabolic syndrome could possibly be confounded by physical activity. However, in our study, even after adjusting for occupational activity or categorized by total activity, we still found metabolic syndrome to be significantly influenced by the time spent viewing TV. This finding suggests TV viewing is an independent risk factor associated with metabolic syndrome, rather than being related to it through the effect of physical activity.

Another mechanism that could explain the effect of TV viewing on metabolic syndrome, could be that physical inactivity would reduce the triglyceride uptake from plasma to muscle and it would further lower serum HDL concentrations due to the decrease of lipoprotein lipase (LPL) activity in the skeletal muscle [48]. In this animal experiment, Hamilton slightly suspended 10 SpragueDawley rats' hind limbs without a weight load for 6 and 12 hours to simulate the physical inactivity. He found that the average LPL activity in rats' skeletal muscles is markedly reduced from 100 percent $(S D, 7)$ to 59 percent (SD, $5)$ in 6 hours and to 12 percent $(S D, 5)$ in 12 hours [48]. This finding supports our conclusion that the length of sedentary time (e.g., TV viewing time) is an independent risk factor for metabolic syndrome.

Even though our study population is fairly large; the study has several limitations. Our study is a cross-sectional design which makes it hard to make conclusions about the causal relationship between TV viewing time and the risk of having metabolic syndrome. Another limitation is that we did not have any information about the length of time spent using a computer and reading time, popular pastimes that also require very little physical activity. All the participants in our study were 40 years, or older and they may not be regular computer users.

\section{Conclusion}

To summarize, we found that excess TV viewing had an adverse effect on metabolic syndrome, a finding that was found to be statistically significant. This result was not affected by physical activity. However, sufficient physical activity is associated with lower prevalence of metabolic syndrome. This implies that not only can it be reduced by an increase in physical activity but also by a decrease in sedentary behavior. A further study needs to elucidate the relationship between different kinds of sedentary behaviors and metabolic syndrome.

\section{Competing interests}

The authors declare that they have no competing interests.

\section{Authors' contributions}

C-CL and T-CL contributed equally to the design of the study and direction of its implementation, including supervision of the field activities, quality assurance and control. P-CC, C-SL, C-IL, W-YL, C-CC and S-YY supervise the field activities. $\mathrm{P}-\mathrm{CC}$ and $\mathrm{M}$-TW helped conduct the literature review and prepare the Materials and Methods and the Discussion sections of the text. P-CC, T-CL and C-IL designed the study's analytic strategy and conducted the data analysis.

\section{Acknowledgements}

This study was supported by grants from the National Science Council of Taiwan (NSC93-23।4-B-039-025 \& NSC94-23।4-B-039-024). The authors express their special thanks to the interviewers and nurses who participated in recruiting participants and data collection.

\section{References}

I. Ford ES, Giles WH, Dietz WH: Prevalence of the metabolic syndrome among US adults: findings from the third National Health and Nutrition Examination Survey. JAMA 2002, 287:356-9.

2. Ford ES, Giles WH: A comparison of the prevalence of the metabolic syndrome using two proposed definitions. Diabetes Care 2003, 26:575-8I.

3. Ford ES: Prevalence of the metabolic syndrome in US populations. Endocrinology \& Metabolism Clinics of North America 2003, 33:333-50.

4. Meigs JB, Wilson PW, Nathan DM, D'Agostino RB Sr, Williams K, Haffner SM: Prevalence and characteristics of the metabolic syndrome in the San Antonio Heart and Framingham Offspring Studies. Diabetes 2003, 52:2160-7.

5. Park YW, Zhu S, Palaniappan L, Heshka S, Carnethon MR, Heymsfield $\mathrm{SB}$ : The metabolic syndrome: prevalence and associated risk factor findings in the US population from the Third National Health and Nutrition Examination Survey, 1988-1994. Archives of Internal Medicine 2003, 163:427-36.

6. Chuang SY, Chen CH, Tsai ST, Chou P: Clinical identification of the metabolic syndrome in Kinmen. Acta Cardiol Sin 2002, 18:16-23.

7. Chien-Jen Chen, San-Lin You, Chyi-huey Bai, Ta-Chen Su, Chin-Hsiao Tseng, Kuo-Liong Chien, Lee-Ching Hwang: The prevalence of hypertension, hyperglycemia and hyperlipidemia survey in Taiwan. 2002. Chinese

8. Wen-Yuan, Lin : A Population-based Case-control Proband Study on Familial Aggregation of Metabolic Syndrome. (Chinese). Master dissertation of Institute of Preventive Medicine College of Public Health, National Taiwan University; 2002.

9. Dou XF, Zhang HY, Sun K, Wang DW, Liao YH, Ma AQ, Zhu ZM, Zhao BR, Zhao JZ, Hui RT: Metabolic syndrome strongly linked to stroke in Chinese. (Chinese). Chung-Hua i Hsueh Tsa Chih 2004, 84:539-42.

10. Hanson RL, Imperatore G, Bennett PH, Knowler WC: Components of the "metabolic syndrome" and incidence of type 2 diabetes. Diabetes 2002, 5 I:3120-27.

II. Hu G, Qiao Q, Tuomilehto J, Balkau B, Borch-Johnsen K, Pyorala K: Prevalence of the metabolic syndrome and its relation to allcause and cardiovascular mortality in nondiabetic European men and women. Arch Intern Med 2004, 164:1066-76. 
12. Isomaa B, Almgren P, Tuomi T, Forsén B, Lahti K, Nissén M, Taskinen MR: Cardiovascular morbidity and mortality associated with the metabolic syndrome. Diabetes Care 200I, 24:683-89.

13. Kekalainen P, Sarlund H, Pyorala K, Laakso M: Hyperinsulinemia cluster predicts the development of type 2 diabetes independently of family history of diabetes. Diabetes Care 1999, 22:86-92.

14. Klein BE, Klein R, Lee KE: Components of the metabolic syndrome and risk of cardiovascular disease and diabetes in beaver dam. Diabetes Care 2002, 25:1790-94.

15. Laaksonen DE, Lakka HM, Niskanen LK, Kaplan GA, Salonen JT, Lakka TA: Metabolic syndrome and development of diabetes mellitus: application and validation of recently suggested definitions of the metabolic syndrome in a prospective cohort study. Am J Epidemiol 2002, I 56:1070-77.

16. Lakka HM, Laaksonen DE, Lakka TA, Niskanen LK, Kumpusalo E, Tuomilehto J, Salonen JT: The metabolic syndrome and total and cardiovascular disease mortality in middle-aged men. JAMA 2002, 288:2709-16.

17. Malik S, Wong ND, Franklin SS, Kamath TV, L'Italien GJ, Pio JR, Williams GR: Impact of the metabolic syndrome on mortality from coronary heart disease, cardiovascular disease, and all causes in United States adults. Circulation 2004, I I 0: I245-50.

18. Onat A, Ceyhan K, Basar O, Erer B, Toprak S, Sansoy V: Metabolic syndrome: major impact on coronary risk in a population with low cholesterol levels-a prospective and cross-sectiona evaluation. Atherosclerosis 2002, I 65:285-292.

19. Pyorala M, Miettinen H, Halonen P, Laakso M, Pyorala K: Insulin resistance syndrome predicts the risk of coronary heart disease and stroke in healthy middle-aged men: the 22-year follow-up results of the Helsinki Policemen Study. Arterioscler Thromb Vasc Biol 2000, 20(2):538-44.

20. Resnick HE, Jones K, Ruotolo G, Jain AK, Henderson J, Lu W, Howard BV: Insulin resistance, the metabolic syndrome, and risk of incident cardiovascular disease in nondiabetic American Indians: the Strong Heart Study. Diabetes Care 2003 26:86I-7.

21. Lidfeldt J, Nyberg P, Nerbrand C, Samsioe G, Schersten B, Agardh CD: Socio-demographic and psychosocial factors are associated with features of the metabolic syndrome. The Women's Health in the Lund Area (WHILA) study. Diabetes, Obesity \& Metabolism 2003, 5:106-12.

22. Panagiotakos DB, Pitsavos C, Chrysohoou C, Skoumas J, Tousoulis D, Toutouza M, Toutouzas P, Stefanadis C: Impact of lifestyle habits on the prevalence of the metabolic syndrome among Greek adults from the ATTICA study. American Heart Journal 2004, | 47:106-12.

23. Park HS, Oh SW, Cho SI, Choi WH, Kim YS: The metabolic syndrome and associated lifestyle factors among South Korean adults. International Journal of Epidemiology 2004, 33:328-36

24. Park YW, Zhu S, Palaniappan L, Heshka S, Carnethon MR, Heymsfield SB: The metabolic syndrome: prevalence and associated risk factor findings in the US population from the Third National Health and Nutrition Examination Survey, I988-1994. Archives of Internal Medicine 2003, 163:427-36.

25. Stone NJ: Focus on lifestyle change and the metabolic syndrome. Endocrinology \& Metabolism Clinics of North America 2004, 33:493-508.

26. Wamala SP, Lynch J, Horsten M, Mittleman MA, Schenck-Gustafsson $\mathrm{K}$, Orth-Gomer K: Education and the metabolic syndrome in women. Diabetes Care 1999, 22:1999-2003.

27. Fitzgerald SJ, Kriska AM, Pereira MA, de Courten MP: Associations among physical activity, television watching, and obesity in adult Pima Indians. Med Sci Sports Exerc 1997, 29:910-915.

28. Fung TT, Hu FB, Yu J, Chu NF, Spiegelman D, Tofler GH, Willett WC, Rimm EB: Leisure-time physical activity, television watching, and plasma biomarkers of obesity and cardiovascular disease risk. Am J Epidemiol 2000, I 52: I I7I-78.

29. Hu FB, Leitzmann MF, Stampfer MJ, Colditz GA, Willett WC, Rimm $E B$ : Physical activity and television watching in relation to risk for type $\mathbf{2}$ diabetes mellitus in men. Arch Intern Med 2001, 16 I:1542-48.

30. Hu FB, Li TY, Colditz GA, Willett WC, Manson JE: Television watching and other sedentary behaviors in relation to risk of obesity and type 2 diabetes mellitus in women. JAMA 2003, 289: $|785-9|$.
3I. Jakes RW, Day NE, Khaw KT, Luben R, Oakes S, Welch A, Bingham $\mathrm{S}$, Wareham NJ: Television viewing and low participation in vigorous recreation are independently associated with obesity and markers of cardiovascular disease risk: EPIC-Norfolk population-based study. Eur J Clin Nutr 2003, 57:1089-96.

32. Kronenberg F, Pereira MA, Schmitz MK, Arnett DK, Evenson KR Crapo RO, Jensen RL, Burke GL, Sholinsky P, Ellison RC, Hunt SC: Influence of leisure time physical activity and television watching on atherosclerosis risk factors in the NHLBI Family Heart Study. Atherosclerosis 2000, I 53:433-43.

33. Salmon J, Bauman A, Crawford D, Timperio A, Owen N: The association between television viewing and overweight among Australian adults participating in varying levels of leisuretime physical activity. Int J Obes Relat Metab Disord 2000, 24:600-06.

34. Gustat J, Srinivasan SR, Elkasabany A, Berenson GS: Relation of selfrated measures of physical activity to multiple risk factors of insulin resistance syndrome in young adults: the Bogalusa Heart Study. Journal of Clinica Epidemiology 2002, 55:997-1006.

35. Ford ES, Kohl HW 3rd, Mokdad AH, Ajani UA: Sedentary behavior, physical activity, and the metabolic syndrome among U.S. adults. Obesity Research 2005, I3:608-I4.

36. Bertrais S, Beyeme-Ondoua JP, Czernichow S, Galan P, Hercberg S, Oppert JM: Sedentary behaviors, physical activity, and metabolic syndrome in middle-aged French subjects. Obesity Research 2005, 13:936-44.

37. Dunstan DW, Salmon J, Owen N, Armstrong T, Zimmet PZ, Welborn TA, Cameron AJ, Dwyer T, Jolley D, Shaw JE, AusDiab Steering Committee: Associations of TV viewing and physical activity with the metabolic syndrome in Australian adults. Diabetologia 2005, 48:2254-6I.

38. Gaetano Crepaldi, Stefania Maggi: The metabolic syndrome: a historical context. Diabetes voice 2006, 51 :8-10.

39. Chen CC, Li TC, Li Cl, Liu CS, Lin WY, Wu MT, Lai MM, Lin CC: The Association with Cholesterol Levels in Women. Metabolism 2007, 56:1216-20.

40. Lin CC, Liu CS, Li TC, Chen CC, Li Cl, Lin WY: Microalbuminuria and the metabolic syndrome and its components in the Chinese population. European Journal of Clinical Investigation 2007, 37( 10$)$ :783-90.

4I. Lin CC, Liu CS, Lai MM, Li Cl, Chen CC, Chang PC, Lin WY, Lee YD, Lin T, Li TC: Metabolic Syndrome and Its Associated Risk Factors in a Taiwanese Metropolitan Adult Population. BMC Public Health 2007, 7(I):739-743.

42. Walter Willett, Meir Stampfer J: Total Energy Intake: Implications for Epidemiologic Analyses. Am J Epidemiol 1986, I 24(I): I 7-27

43. Jeffery RW, French SA: Epidemic obesity in the United States: are fast foods and television viewing contributing? Am J Public Health 1998, 88:277-80.

44. Carroll $S$, Dudfield $M$ : What is the relationship between exercise and metabolic abnormalities? A review of the metabolic syndrome. Sports Med 2004, 34(6):37|-4I8.

45. Eriksson J, Taimela S, Koivisto VA: Exercise and the metabolic syndrome. Diabetologia 1997, 40:125-35

46. Irwin ML, Ainsworth BE, Mayer-Davis El, Addy CL, Pate RR, Durstine JL: Physical activity and the metabolic syndrome in a tri-ethnic sample of women. Obesity Research 2002, 10:1030-7.

47. Rennie KL, McCarthy N, Yazdgerdi S, Marmot M, Brunner E: Association of the metabolic syndrome with both vigorous and moderate physical activity. International Journal of Epidemiology 2003, 32:600-6.

48. Bey L, Hamilton MT: Suppression of skeletal muscle lipoprotein lipase activity during physical inactivity: a molecular reason to maintain daily low-intensity activity. Journal of Physiology 2003, 55 I :673-82.

\section{Pre-publication history}

The pre-publication history for this paper can be accessed here:

http://www.biomedcentral.com/1471-2458/8/193/pre pub 